\title{
Maladies d'origine alimentaire et hydrique dans les populations canadiennes autochtones: examen de la portée
}

\author{
JKH Jung ${ }^{1}$, K Skinner ${ }^{1 *}$
}

\section{Résumé}

Contexte : Les populations autochtones présentent souvent un risque plus élevé de contracter des maladies d'origine alimentaire que la population canadienne générale.

Objectif : Étudier l'ensemble de la documentation concernant le lien entre la salubrité des aliments et la fréquence des maladies d'origine alimentaire et hydrique dans les populations canadiennes autochtones.

Méthodologie : Un examen de la portée a été effectué à l'aide de chaînes de recherche dans cinq bases de données et dans la littérature grise afin de repérer tous les articles ayant étudié une population canadienne autochtone et ayant cité de possibles associations entre des pratiques de salubrité des aliments (y compris la consommation et la préparation d'aliments traditionnels et d'aliments vendus au détail) ou de sécurité de l'eau et des maladies d'origine alimentaire ou hydrique. Deux auteurs ont sélectionné les articles en fonction de critères $d$ 'inclusion et d'exclusion. Les documents inclus ont été analysés pour y repérer des thèmes émergents.

Résultats : Sur 1718 documents uniques identifiés, 21 documents ont été sélectionnés. Les maladies d'origine alimentaire étaient les plus fréquentes chez les enfants de 14 ans et moins. Le morse, le phoque, le caribou et la baleine étaient les aliments traditionnels les plus couramment porteurs de maladies d'origine alimentaire et étaient essentiellement associés au botulisme et à la trichinose. Outre la consommation d'aliments crus, la fermentation était la méthode de préparation traditionnelle la plus fréquemment associée aux maladies d'origine alimentaire. La salubrité des aliments vendus au détail était source de préoccupation, mais aucun lien clair n'a été établi avec une maladie d'origine alimentaire. Enfin, bien que l'eau du robinet ait été source d'inquiétude, I'utilisation d'autres sources d'eau, comme l'eau non traitée des ruisseaux, ainsi que les pratiques d'hygiène et de nettoyage dans les communautés pour lesquelles des avis d'ébullition d'eau ont été émis, étaient les comportements à risque les plus couramment associés aux maladies d'origine hydrique.

Conclusion : La consommation de certaines viandes de gibier et l'utilisation de méthodes de fermentation traditionnelles peuvent augmenter le risque de maladies d'origine alimentaire chez les populations autochtones. Des inquiétudes concernant l'eau du robinet peuvent entraîner l'utilisation d'autres sources d'eau non salubres. Des recherches supplémentaires sont nécessaires pour étudier des aliments possibles culturellement adaptés et des possibilités de consommation d'eau salubre.
Affiliation

${ }^{1}$ School of Public Health and Health Systems, Université de Waterloo, Waterloo (Ontario)

*Correspondance : kskinner@ uwaterloo.ca

Citation proposée : Jung JKH, Skinner K. Maladies d'origine alimentaire et hydrique dans les populations canadiennes autochtones : examen de la portée. Relevé des maladies transmissibles au Canada.

2017;43(1):7-14. https://doi.org/10.14745/ccdr.v43i01a02f

\section{Introduction}

Les maladies d'origine alimentaire et hydrique constituent des problèmes de santé publique importants dans le monde entier, leur morbidité et leur mortalité touchant à la fois les pays développés et les pays en développement $(1,2)$. Essentiellement causées par des bactéries, des virus et des parasites, les maladies d'origine alimentaire et hydrique prennent généralement la forme de symptômes gastro-intestinaux $(3,4)$. Au Canada, on estime que les maladies d'origine alimentaire touchent environ une personne sur huit (quatre millions de cas) chaque année (3). Toutefois, le fardeau des maladies $d^{\prime}$ 'origine alimentaire n'est pas réparti équitablement, car dans de nombreuses communautés autochtones, le risque de maladie entérique est considéré comme supérieur à la moyenne nationale (5). De plus, dans ces communautés, le risque de 
maladie d'origine hydrique est également plus élevé, notamment en raison de facteurs environnementaux (6).

Ce risque plus élevé de maladies d'origine alimentaire et hydrique dans les communautés autochtones s'explique par plusieurs raisons. Une grande proportion d'autochtones appartient aux groupes les plus à risque : nourrissons, jeunes enfants, femmes enceintes et personnes âgées (7). Toutefois, cette prévalence plus élevée peut également être associée aux méthodes de préparation et à la consommation d'aliments traditionnels. Ainsi, la consommation de viande crue (par exemple le phoque, la baleine, le morse et le caribou) est fréquente dans certains groupes autochtones, et cela présente un risque pour la santé en raison de la présence de pathogènes normalement détruits par une cuisson adaptée (7). Le non-respect des avis d'ébullition d'eau émis pour les communautés autochtones canadiennes peut également avoir des répercussions sur le risque de contracter une maladie d'origine hydrique $(5,8,9)$.

À notre connaissance, aucun examen complet des maladies d'origine alimentaire et hydrique causées par des pratiques en matière de salubrité des aliments et de l'eau dans les populations canadiennes autochtones n'a été effectué. Ainsi, un examen de la portée a été réalisé, car ce type d'examen est particulièrement utile en l'absence d'examen complet. L'objectif de cet examen de la portée était d'étudier l'étendue, la nature et la variété des études concernant le lien entre salubrité des aliments et fréquence des maladies d'origine alimentaire et hydrique dans les populations canadiennes autochtones.

\section{Méthodologie}

Cet examen de la portée a suivi le cadre en cinq étapes élaboré par Arksey et O'Malley (10). Tout d'abord, nous avons défini la question de recherche : "Que sait-on actuellement sur le lien entre la salubrité des aliments et la fréquence des maladies d'origine alimentaire/hydrique dans la population canadienne autochtone? " La salubrité des aliments était définie comme la manipulation, la préparation et le stockage d'aliments afin de préserver leur qualité et d'éviter toute contamination (11). La préparation et la consommation d'aliments traditionnels étaient également incluses. Les aliments traditionnels sont ceux obtenus à partir de plantes ou d'animaux locaux, par la cueillette ou la récolte, possédant une signification culturelle (12) et généralement synonymes des termes « aliments prélevés dans la nature » et " aliments issus de la flore et de la faune sauvage ». Nous avons inclus le risque de maladie d'origine hydrique s'il était associé à des pratiques individuelles/communautaires, et pas uniquement à une contamination environnementale. Nous avons pris en compte toutes les formes de maladies causées par des aliments ou des sources d'eau contaminées, et pas simplement les formes associées à des maladies gastro-intestinales aiguës (par exemple nous avons inclus I'hépatite A dans notre recherche). Nous avons choisi le terme " autochtones " pour désigner les populations des Premières Nations, des Métis et des Inuits. Toutefois, les groupes ont également été désignés par les termes utilisés dans les études.

\section{Identification des études}

Une bibliothécaire de recherche a guidé l'élaboration des chaînes de recherche pertinentes pour cinq bases de données universitaires (Annexe). En raison de la définition très large de "salubrité des aliments", ce terme n'a pas été inclus dans la stratégie de recherche, mais il a été utilisé dans le cadre des critères d'inclusion et d'exclusion (Encadré). Des recherches manuelles ont également été effectuées dans des revues (non répertoriées dans les bases de données) et dans les publications de certains auteurs ayant étudié la consommation d'aliments dans les populations autochtones (Annexe). Toutes les citations obtenues ont été exportées vers RefWorks@.

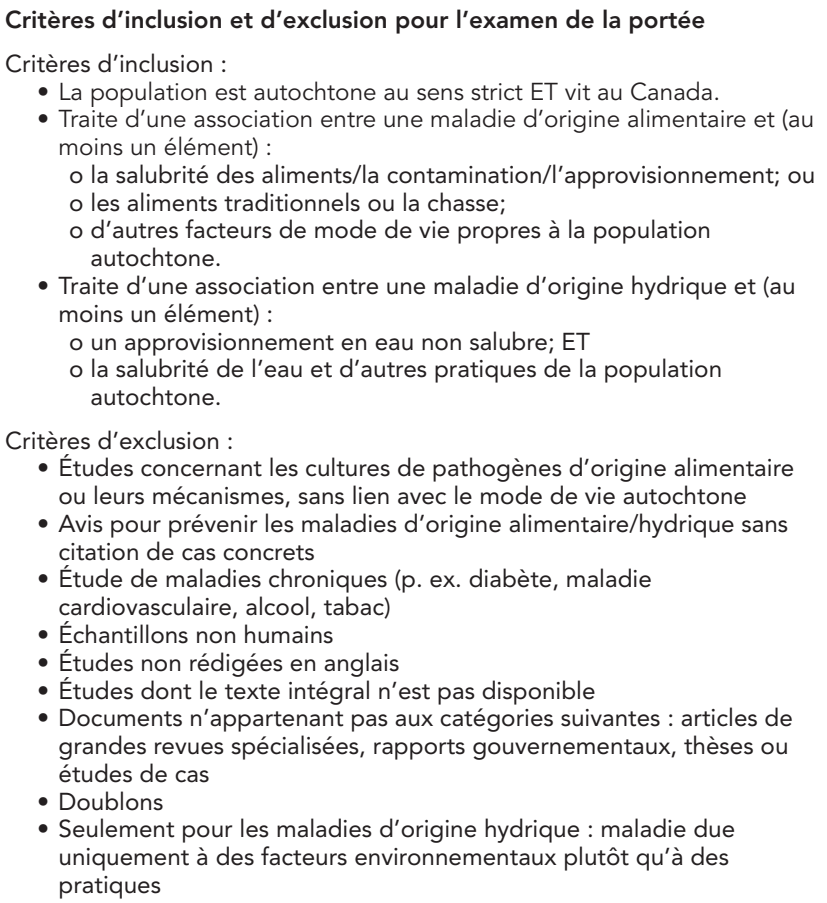

- Étude de maladies chroniques (p. ex. diabète, maladie cardiovasculaire, alcool, tabac)

- Échantillons non humains

- Études non rédigées en anglais

- Études dont le texte intégral n'est pas disponible

- Documents n'appartenant pas aux catégories suivantes : articles de grandes revues spécialisées, rapports gouvernementaux, thèses ou études de cas

- Doublons

- Seulement pour les maladies d'origine hydrique : maladie due uniquement à des facteurs environnementaux plutôt qu'à des pratiques

Pour accéder à la littérature grise, un moteur de recherche Google personnalisé intégrant les sites Web des agences de la santé publique et des ministères de la santé fédéraux et provinciaux du Canada a été utilisé $(13,14)$. Une base de données des mémoires et thèses a également été utilisée ainsi que trois sites Web du gouvernement fédéral (Annexe). Dans la littérature grise, plusieurs recherches ont été effectuées à l'aide de différentes associations de termes clés plutôt que d'une chaîne de recherche systématique (12; Annexe).

\section{Sélection de l'étude}

Les titres et les résumés de toutes les citations obtenues ont été analysés en fonction des critères a priori d'inclusion/d'exclusion. Deux examinateurs indépendants ont analysé le texte intégral des documents à l'aide des critères pour l'inclusion finale. Les conflits faisaient l'objet d'une discussion entre les examinateurs et les critères étaient revus jusqu'à ce qu'un accord soit trouvé. Le kappa de Cohen $(k)$ a été utilisé pour évaluer l'accord entre les examinateurs. Une valeur $k$ égale à 0,7 était considérée comme suffisante. Aucune limite n'a été fixée concernant l'année 
de publication. En cas de documents en double pour la même étude (publications multiples à partir des mêmes données) ou pour le même rapport (version révisée d'un rapport d'une année précédente), le document contenant les renseignements les plus pertinents a été sélectionné.

\section{Collecte, analyse et soumission des données}

Pour chaque document, les renseignements recueillis étaient les suivants : auteur(s), année de publication, lieu de l'étude au Canada, population autochtone spécifique, type de document, objectif de l'étude, nombre de cas, type de maladie traitée, pathogène spécifique traité et principaux effets signalés sur la santé. Les résultats ont été résumés sous forme d'analyse thématique qualitative et de thèmes émergents. Des variables démographiques telles que l'âge, le sexe et les facteurs communautaires ont été étudiées afin d'établir de possibles liens avec des maladies d'origine alimentaire et hydrique. Les types d'aliments traditionnels particuliers consommés par les personnes tombées malades ainsi que les méthodes de préparation associées aux maladies ont été signalés. Le rôle des aliments vendus au détail (ou achetés) dans les communautés autochtones et pouvant augmenter le risque de maladie a été étudié, et toutes les pratiques en matière de salubrité de l'eau associées à une maladie d'origine hydrique ont été signalées. Comme pour la plupart des examens de la portée, aucune évaluation formelle de la qualité des études n'a été réalisée (9).

\section{Résultats}

Sur les 1718 documents uniques identifiés dans les bases de données, par les recherches manuelles et dans la littérature grise, 21 documents (20 articles de revue et un rapport) ont été inclus dans l'analyse qualitative (figure 1). Les raisons des exclusions sont indiquées dans la figure 1. Au niveau de l'analyse du texte intégral des articles, l'accord entre les examinateurs était plutôt fort $(k=0,75)$.

\section{Facteurs démographiques et sociaux}

Sur les 21 documents examinés, huit (38\%) étudiaient la corrélation entre l'âge et la fréquence des maladies d'origine alimentaire et hydrique $(7,8,19,25,28-31)$. Sur ces huit documents, six (75\%) indiquaient une prévalence plus élevée chez les enfants de 14 ans et moins $(7,19,28-31)$. Une étude ne signalait aucune différence en fonction de l'âge (8), et une étude montrait que la plupart des cas de trichinose concernaient des personnes de plus de 60 ans, le plus souvent de sexe féminin (25). Sur les six documents étudiant une corrélation avec le sexe, la moitié indiquaient une distribution à peu près équivalente pour le botulisme (16), E. coli (28) et l'hépatite A $(30,31)$. Le surpeuplement des habitations était signalé dans quatre articles, notamment en hiver $(5,29-31)$. Deux études $(8,29)$ signalaient que des réseaux sociaux étroits dans de petites communautés isolées pouvaient constituer un autre mode possible de transmission des maladies d'origine alimentaire. Enfin, un document (7) suggérait que la pratique du partage de nourriture avec les membres de la famille ou de la communauté, après une chasse ou une récolte, pouvait être un véhicule de transmission de maladies d'origine alimentaire.
Figure 1 : organigramme du processus d'analyse

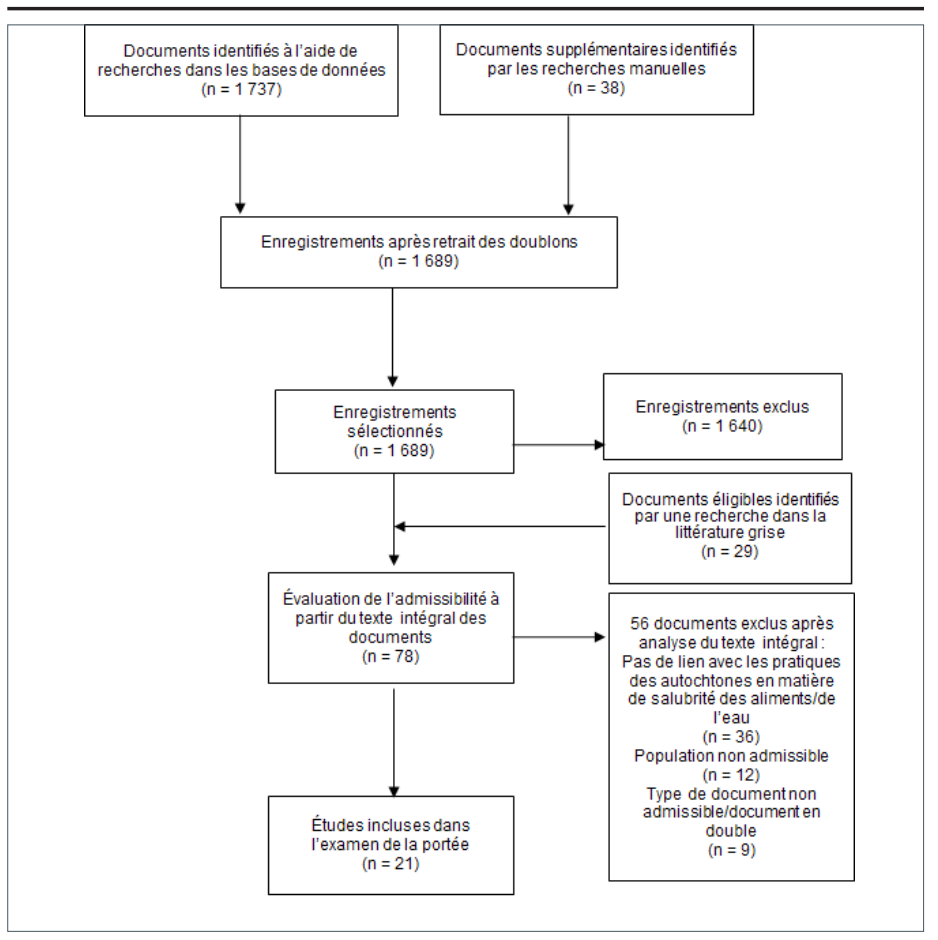

\section{Maladies d'origine alimentaire}

Seize études concernaient les maladies d'origine alimentaire (tableau 1). Sur ces 16 études, 15 (94\%) portaient sur des populations inuites, et les Premières Nations de Colombie-Britannique étaient les plus fréquemment étudiées ensuite. Les Territoires du Nord-Ouest étaient les plus étudiés (50\%); les autres provinces et territoires étudiés étaient le Nunavut, la Colombie-Britannique, le Québec, I'Ontario et le Manitoba.

\section{Types d'aliments traditionnels et méthodes de préparation traditionnelles}

Les principaux thèmes traités par les études sont indiqués dans la figure 2. Sur les 18 études pertinentes, le morse (44\%), le phoque (39\%), le caribou (39\%) et la baleine (33\%) étaient les aliments traditionnels les plus souvent associés à des maladies d'origine alimentaire, notamment lorsqu'ils étaient consommés crus. Les autres aliments traditionnels associés à des maladies étaient les œufs de saumon fermentés $(7,16,21-23)$ et le poisson comme l'omble, le saumon et la truite $(5,7,21)$. Outre la consommation d'aliments crus, la fermentation d'aliments traditionnels était la méthode de préparation traditionnelle la plus fréquemment associée aux maladies d'origine alimentaire $(7,16,20-23,25,26)$. Cette méthode était le plus souvent utilisée avec le phoque $(7,20-23)$, le morse $(7,20,25,26)$ et les œufs de saumon fermentés $(7,16,19,20)$. Une étude a souligné que les méthodes de fermentation utilisées par les Premières Nations et les Inuits ne produisaient pas d'acide lactique, d'acide acétique ou d'éthanol pour inhiber la croissance de pathogènes (7). Les méthodes de fermentation autochtones traditionnelles ont été décrites comme un processus de décomposition ou de 
Tableau 1 : Études canadiennes sur les maladies d'origine alimentaire dans les populations autochtones

\begin{tabular}{|c|c|}
\hline $\begin{array}{l}\text { Population et emplacement } \\
\text { (Réf.) }\end{array}$ & $\begin{array}{l}\text { Objectif de l'étude } \\
\text { (nombre de cas) }\end{array}$ \\
\hline $\begin{array}{l}\text { Inuits au Labrador et au Nunavut } \\
(5)^{1}\end{array}$ & $\begin{array}{l}\text { Estimer le fardeau des maladies } \\
\text { gastro-intestinales aiguës autodéclarées à l'échelle } \\
\text { de la communauté dans les communautés inuites de } \\
\text { Rigolet, au Labrador }(n=30) \text { et d'Iqaluit au Nunavut } \\
(\mathrm{n}=72)\end{array}$ \\
\hline $\begin{array}{l}\text { Inuits, Premières Nations, Métis } \\
\text { au Canada }(7)^{1}\end{array}$ & $\begin{array}{l}\text { Identifier les problèmes de salubrité associés } \\
\text { aux aliments traditionnels et aux facteurs } \\
\text { environnementaux, et évaluer l'efficacité des } \\
\text { programmes visant à réduire le nombre de maladies } \\
\text { d'origine alimentaire }\end{array}$ \\
\hline Inuits au Labrador (8) ${ }^{1}$ & $\begin{array}{l}\text { Comprendre l'expérience vécue de maladies gastro- } \\
\text { intestinales aiguës dans une petite communauté } \\
\text { inuite de Rigolet au Canada }(n=30)\end{array}$ \\
\hline $\begin{array}{l}\text { Premières Nations au Manitoba } \\
\text { et Inuits dans les Territoires du } \\
\text { Nord-Ouest (9) }\end{array}$ & $\begin{array}{l}\text { Examiner les causes de diarrhées à Winnipeg et à } \\
\text { Berens River, au Manitoba et à Eskimo Point, dans les } \\
\text { Territoires du Nord-Ouest }(n=172)\end{array}$ \\
\hline $\begin{array}{l}\text { Inuks dans I'Inuvialuit, Territoires } \\
\text { du Nord-Ouest (15) }\end{array}$ & $\begin{array}{l}\text { Identifier les causes du botulisme chez une femme } \\
\text { inuvialuit de } 58 \text { ans }(n=1)\end{array}$ \\
\hline $\begin{array}{l}\text { Inuits au Nunavik (Québec) et } \\
\text { Premières Nations en Colombie- } \\
\text { Britannique (16) }\end{array}$ & $\begin{array}{l}\text { Résumer les cas de botulisme au Canada de } 1985 \\
\text { à } 2005(n=205)\end{array}$ \\
\hline $\begin{array}{l}\text { Inuits, Premières Nations, et } \\
\text { Métis dans les Territoires du } \\
\text { Nord-Ouest (17) }\end{array}$ & $\begin{array}{l}\text { Examiner les facteurs de risques communautaires } \\
\text { pour les maladies gastro-intestinales à déclaration } \\
\text { obligatoire dans les Territoires du Nord-Ouest } \\
\text { ( } n=708)\end{array}$ \\
\hline Inuits au Nunavik, Québec (18) & $\begin{array}{l}\text { Examiner l'efficacité du Programme de prévention de } \\
\text { la trichinellose au Nunavik }(n=95)\end{array}$ \\
\hline Inuits au Nunavut (19) & $\begin{array}{l}\text { Décrire les cas de brucellose et l'examen } \\
\text { bactériologique des organismes isolés }(n=7)\end{array}$ \\
\hline Inuits au Nunavik, Québec (20) & $\begin{array}{l}\text { Résumer quatre éclosions de botulisme non liées } \\
\text { dans la baie d'Ungava au Nunavik, Québec }(n=9)\end{array}$ \\
\hline $\begin{array}{l}\text { Principalement des Inuits au } \\
\text { Québec, dans les Territoires du } \\
\text { Nord-Ouest et en Colombie- } \\
\text { Britannique (21) }\end{array}$ & $\begin{array}{l}\text { Résumer les cas de botulisme au Canada de } 1971 \\
\text { à } 1984(n=113)\end{array}$ \\
\hline $\begin{array}{l}\text { Premières Nations de Colombie- } \\
\text { Britannique et de l'Ontario, et } \\
\text { Inuits au Québec et dans les } \\
\text { Territoires du } \\
\text { Nord-Ouest (22) }\end{array}$ & $\begin{array}{l}\text { Résumer les cas de botulisme au Canada de } 1971 \\
\text { à } 1974(n=42)\end{array}$ \\
\hline $\begin{array}{l}\text { Premières Nations de Colombie- } \\
\text { Britannique et de l'Ontario, et } \\
\text { Inuits au Québec et dans les } \\
\text { Territoires du Nord-Ouest (23) }\end{array}$ & $\begin{array}{l}\text { Résumer les cas de botulisme au Canada de } 1919 \\
\text { à } 1973(n=122)\end{array}$ \\
\hline $\begin{array}{l}\text { Inuits au Nunavut et au Québec } \\
\text { (24) }\end{array}$ & $\begin{array}{l}\text { Présenter trois éclosions de botulisme à Cape Dorset } \\
\text { et dans la baie de Frobisher, au Nunavut; et dans la } \\
\text { baie Wakeham, au Québec chez les Inuits de } 1967 \text { à } \\
1969(n=9)\end{array}$ \\
\hline Inuits au Nunavut (25) & $\begin{array}{l}\text { Décrire une éclosion de trichinellose sur l'île de Baffin } \\
\text { en août-septembre } 1999(n=34)\end{array}$ \\
\hline Inuits au Québec (26) & $\begin{array}{l}\text { Décrire une éclosion de trichinose après la } \\
\text { consommation de viande de morse crue chez } \\
10 \text { habitants inuits de Salluit, au Québec. }(n=10)\end{array}$ \\
\hline $\begin{array}{l}\text { Inuits dans les Territoires du } \\
\text { Nord-Ouest (27) }\end{array}$ & $\begin{array}{l}\text { Examiner les causes de brucellose chez un jeune } \\
\text { garçon inuit âgé de neuf ans }(n=1)\end{array}$ \\
\hline $\begin{array}{l}\text { Inuits dans les Territoires du } \\
\text { Nord-Ouest (28) }\end{array}$ & $\begin{array}{l}\text { Décrire les caractéristiques cliniques et } \\
\text { épidémiologiques d'une éclosion d'Escherichia coli } \\
\text { produisant de la vérotoxine associée à des diarrhées } \\
\text { dans le Keewatin, Territoires du Nord-Ouest }(n=152)\end{array}$ \\
\hline $\begin{array}{l}\text { Inuits dans les Territoires du } \\
\text { Nord-Ouest (29) }\end{array}$ & $\begin{array}{l}\text { Évaluer les facteurs de risques du syndrome } \\
\text { hémolytique et urémique et de gastroentérite chez } \\
\text { les enfants pendant une épidémie d'E. coli O157:H7 } \\
\text { à Arviat, au Nunavut }(n=84)\end{array}$ \\
\hline $\begin{array}{l}\text { Premières Nations de Colombie- } \\
\text { Britannique }(30)^{2}\end{array}$ & $\begin{array}{l}\text { Déterminer si l'incidence de l'hépatite A est plus } \\
\text { élevée dans la population autochtone que dans la } \\
\text { population totale de la Colombie-Britannique et si } \\
\text { c'est associé à la pauvreté et à des conditions de vie } \\
\text { insalubres ( } \mathrm{n}=2 \text { 933) }\end{array}$ \\
\hline $\begin{array}{l}\text { Premières Nations du nord de la } \\
\text { Colombie-Britannique }(31)^{2}\end{array}$ & $\begin{array}{l}\text { Décrire l'éclosion d'hépatite A dans la région } \\
\text { sanitaire Northern Interior de Colombie-Britannique } \\
\text { et l'intervention en santé publique }(n=23)\end{array}$ \\
\hline
\end{tabular}

putréfaction et il est possible que le $\mathrm{pH}$ faible nécessaire pour inhiber la croissance des pathogènes ne soit pas atteint (21).

Figure 2 : Thèmes associés à la salubrité des aliments et de l'eau identifiés dans les études incluses $(n=21)$

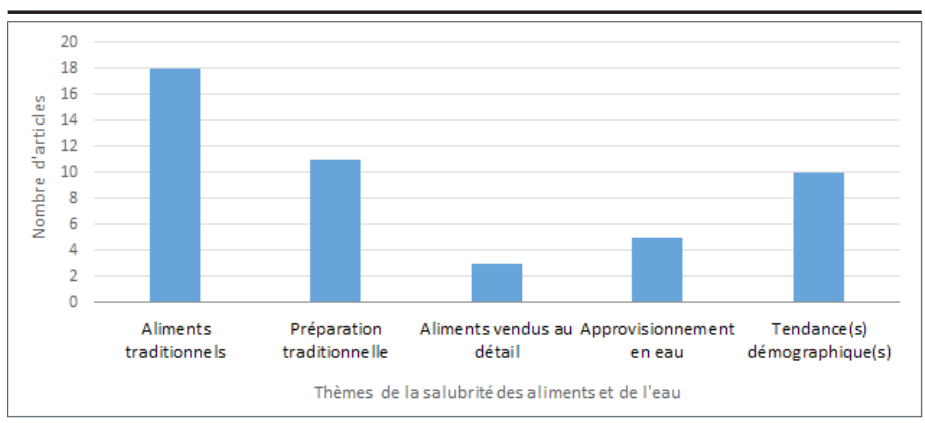

\section{Des types d'infection}

Les types d'infection les plus fréquents sont indiqués dans la figure 3. Près d'un tiers des articles $(n=7)$ traitaient du botulisme $(15,16,20,24)$. La trichinose était traitée par près de $15 \%$ des études. La brucellose, E. coli et l'hépatite A étaient identifiées dans environ $10 \%$ des études. La brucellose était particulièrement associée à la consommation de caribou $(19,27)$.

\section{Figure 3 : Origines courantes des maladies gastro-intestinales identifiées dans les études incluses $(n=21)$}

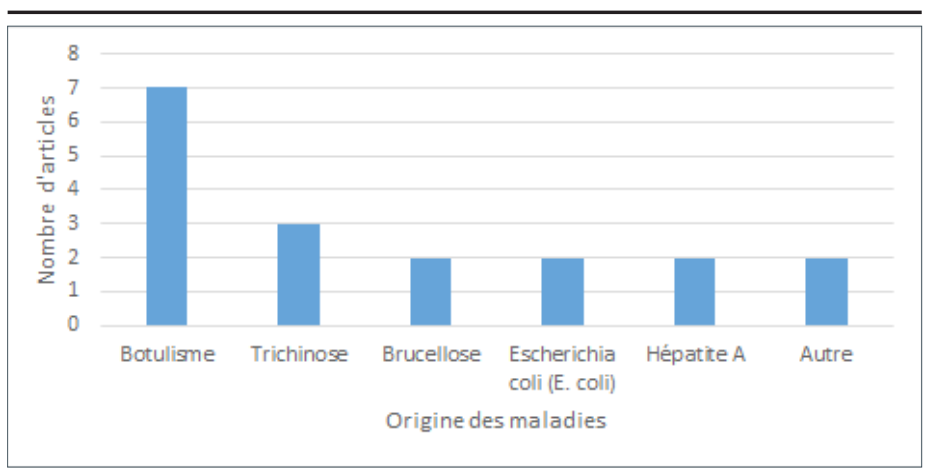

\section{Aliments vendus au détail}

Lors de l'examen de la salubrité des aliments et des aliments autochtones traditionnels, certaines études ont montré que les Autochtones étaient préoccupés par la salubrité des aliments vendus au détail, mais peu d'études ont évalué la corrélation entre les aliments vendus au détail et les maladies d'origine alimentaire dans les populations autochtones (7). Une étude a montré que les aliments vendus au détail pouvaient constituer un facteur de maladie gastro-intestinale aiguë dans une communauté inuite (8), et une autre étude a découvert que le risque de développer une maladie gastro-intestinale aiguë était plus élevé si la personne chargée de la préparation des aliments était employée (5). Les auteurs ont suggéré que les personnes disposant de revenus plus élevés avaient moins de temps pour accéder aux aliments traditionnels, et consommaient donc davantage d'aliments vendus au détail, mais cela n'a pas été confirmé. À l'inverse, une étude menée dans les Territoires du Nord-Ouest (17) a montré que plus les prix des aliments dans les communautés autochtones étaient élevés, plus le risque de 
campylobactériose était faible. Les auteurs pensaient que les prix élevés des aliments entraînaient une baisse de la consommation de viande, de produits laitiers, et de fruits et légumes vendus au détail, les habitants choisissant plutôt d'acheter des produits transformés, ce qui réduisait l'exposition aux pathogènes le plus souvent présents dans les denrées périssables.

\section{Maladies d'origine hydrique}

Le tableau 1 montre qu'environ un quart des études traitaient de pratiques non sécuritaires liées à l'eau dans les communautés autochtones associées à des maladies d'origine alimentaire ou hydrique $(5,7,8,30,32)$. Trois documents $(5,7,8)$ expliquaient que les communautés autochtones préféraient boire de l'eau provenant de sources autres que le robinet, par exemple de l'eau en bouteille ou de l'eau de ruisseau non traitée. Un article (8) précisait que des Inuits de Rigolet, au Labrador, perçoivent l'eau du robinet comme un facteur de risque possible de maladie gastro-intestinale aiguë, alors que selon eux, l'eau de ruisseau non traitée est salubre. Pourtant, dans une étude semblable (5) concernant les mêmes Inuits de Rigolet, la consommation d'eau provenant de sources autres que le robinet était associée au développement de maladies gastro-intestinales aiguës. Le non-respect des avis d'ébullition d'eau, facteur contributif possible de développement de maladies d'origine hydrique, était également une source de préoccupation dans deux études $(8,31)$. Enfin, un article (30) soulignait que les problèmes d'approvisionnement en eau des communautés (qui entraînaient une incidence plus élevée de l'hépatite $A$ dans cette étude) pouvaient également être la cause de lavages de main peu fréquents ou d'un nettoyage inadapté, car la qualité de l'eau est perçue comme non fiable. Ces comportements peuvent à leur tour constituer des vecteurs supplémentaires de la maladie.

\section{Discussion}

Cet article propose l'examen le plus à jour des études concernant la salubrité des aliments et les pratiques non sécuritaires liées à l'eau dans les populations autochtones du Canada entraînant des maladies d'origine alimentaire et hydrique. La plupart des études signalaient que les maladies d'origine alimentaire concernaient essentiellement des enfants âgés de 14 ans et moins et des femmes, et que les logements surpeuplés et le partage de nourriture pouvaient être des véhicules de transmission des maladies. Le morse, le phoque, le caribou et la baleine, notamment consommés crus ou fermentés à l'aide de processus n'inhibant pas la croissance des pathogènes, étaient associés à un risque plus élevé de maladie d'origine alimentaire.

Quelques limites doivent être prises en compte pour l'examen des résultats. Tout d'abord, l'examen n'a identifié que 21 études réalisées au cours des 50 dernières années. Des erreurs ou des biais ont pu intervenir dans le processus de sélection, et certains articles n'ont peut-être pas été repérés ou ont peut-être été inclus dans l'examen ou exclus de celui-ci par erreur. En outre, la recherche était limitée aux documents rédigés en anglais. Nous avons identifié des études signalant l'idée que la consommation d'aliments vendus au détail était associée à des maladies d'origine alimentaire, mais il n'existe actuellement aucune preuve à cet effet. Ce point mérite donc des études supplémentaires. De même, des craintes quant à l'eau du robinet ont été signalées, et bien qu'un problème ait été constaté dans certaines communautés autochtones (32), il n'est pas certain que l'eau du robinet soit associée à des maladies dans toutes les communautés. II semble que le risque associé à la consommation d'eau de ruisseau non traitée soit sous-estimé. Ce point mérite d'être étudié davantage.

\section{Recherche future}

Globalement, les populations autochtones au Canada sont confrontées à des sources d'infection uniques en raison de facteurs environnementaux et sociaux. Des recherches complémentaires doivent être menées pour mieux comprendre ces enjeux et pour déterminer si une approche de santé publique différente est nécessaire pour une prévention efficace. Lorsque l'on cherche des moyens de réduire l'incidence des maladies d'origine alimentaire et les pratiques non sécuritaires liées à l'eau dans les communautés autochtones, les facteurs culturels doivent être pris en compte. Par exemple, il est important de reconnaître que, même si les maladies d'origine alimentaire sont associées à la consommation d'aliments traditionnels, ces aliments présentent également de nombreux bénéfices pour la santé et sont essentiels au bien-être. La préparation et la consommation d'aliments traditionnels aident à renforcer la culture et l'identité autochtones (33) et contribuent au régime alimentaire global, car ils sont riches en fer, en zinc et en protéines (34). De même, bien que le partage de nourriture ait été associé à des infections d'origine alimentaire, les populations autochtones accordent de l'importance aux liens sociaux qui apportent de nombreux bénéfices à leur santé, à leur bien-être, à leur spiritualité et à l'esprit communautaire. Tous ces facteurs doivent être soigneusement pris en compte lors de l'élaboration de lignes directrices sur la salubrité des aliments. Les programmes ont plus de chance d'être efficaces s'ils sont conçus avec l'aide des communautés et dans le respect des systèmes de connaissance autochtones et des pratiques culturelles concernant les aliments (7). Ainsi, le gouvernement du Nunavut a conçu des lignes directrices pour la salubrité des aliments s'appliquant à la nourriture traditionnelle servie dans les installations et les programmes communautaires financés par le gouvernement (35). Ces lignes directrices pourraient être prises en compte et appliquées dans d'autres contextes.

L'influence des changements climatiques sur les maladies d'origine alimentaire dans les communautés autochtones et leurs répercussions sur le système de soins de santé méritent d'être examinées davantage. Ceci est particulièrement vrai, car des températures plus élevées peuvent entraîner une hausse des maladies d'origine alimentaire sensibles à la température comme le botulisme $(7,36)$ qui peuvent à leur tour créer des coûts financiers importants pour le système de soins de santé.

\section{Conclusion}

Il existe peu de recherches examinant les difficultés uniques relatives à la salubrité des aliments et de l'eau auxquelles les populations canadiennes autochtones doivent faire face et qui pourraient être associées à leur environnement, aux aliments traditionnels, aux techniques de préparation des aliments ainsi qu'à des croyances et à des pratiques sociales et culturelles. II semble que la consommation de certaines viandes de gibier et 
I'utilisation de méthodes de fermentation traditionnelles peuvent augmenter le risque de maladies d'origine alimentaire chez les populations autochtones. Des recherches supplémentaires sont nécessaires pour identifier des pratiques culturellement adaptées assurant la salubrité des aliments.

\section{Remerciements}

Les auteurs aimeraient remercier Jackie Stapleton, la bibliothécaire universitaire de l'École de santé publique et des systèmes de santé de l'Université de Waterloo, pour les avoir aidés à élaborer les chaînes de recherche et à établir une méthodologie de sélection adaptée.

\section{Conflit d'intérêts}

Aucun.

\section{Financement}

Aucun.

\section{Références}

1. Newell DG, Koopmans M, Verhoef L, Duizer E, Aidara-Kane A, Sprong H, et al. Foodborne diseases: The challenges of 20 years ago still persist while new ones continue to emerge. Int J Food Microbiol. 2010;139 Suppl 1:S3-15.

2. Thomas MK, Majowicz SE, Pollari F, Sockett PN. Le fardeau des maladies gastro-intestinales aiguë au Canada, 19992007: résumé provisoires des activités relatives à I'ENMGA. Relevé des maladies transmissibles au Canada. 2008;34(5):815. Disponible à l'adresse : http://www.phac-aspc.gc.ca/ publicat/ccdr-rmtc/08vol34/dr-rm3405b-fra.php.

3. Thomas MK, Murray R, Flockhart L, Pintar K, Pollari F, Fazil A, et al. Estimates of the burden of foodborne illness in Canada for 30 specified pathogens and unspecified agents, circa 2006. Foodborne Pathog Dis. 2013;10(7):639-48.

4. Agence de la santé publique au Canada. Rapport de I'administrateur en chef de la santé publique sur l'état de la santé publique au Canada, 2013. Les maladies infectieuses — Une menace perpétuelle. Ottawa :ASPC; 2013 [Internet] [consulté le 20 sept 2016]. Disponible à l'adresse : http:// www.phac-aspc.gc.ca/cphorsphc-respcacsp/2013/foodwater_alim-eau-fra.php.

5. Harper SL, Edge VL, Ford J, Thomas MK, Pearl DL, Shirley J, et al. Acute gastrointestinal illness in two Inuit communities: Burden of illness in Rigolet and lqaluit, Canada. Epidemiol Infect. 2015;143(14):3048-63.

6. Harper SL, Edge VL, Schuster-Wallace CJ, Berke O, McEwen SA. Weather, water quality and infectious gastrointestinal illness in two Inuit communities in Nunatsiavut, Canada: Potential implications for climate change. Ecohealth. 2011;8(1):93-108.
7. Food Safety Network. Safe preparation and storage of Aboriginal traditional/country foods: A review. Aston Manor SA: Food Safety Network; 2009.

8. Harper SL, Edge VL, Ford J, Thomas MK, IHACC Research Group, Rigolet Inuit Community Government, et al. Lived experience of acute gastrointestinal illness in Rigolet, Nunatsiavut: "just suffer through it". Soc Sci Med. 2015;126:86-98.

9. Gurwith M, Wenman W, Gurwith D, Brunton J, Feltham S, Greenberg $\mathrm{H}$. Diarrhea among infants and young children in Canada: A longitudinal study in three northern communities. J Infect Dis. 1983;147(4):685-92.

10. Arksey H, O'Malley L. Scoping studies: Towards a methodological framework. Int J Soc Res Methodol. 2005;8(1):19-32.

11. University of Maryland Medical Center [Internet]. Food safety. Baltimore MD: University of Maryland; 2014 [mis à jour le 13 juin 2016; consulté le 13 juin 2016]. Disponible à l'adresse : http://umm.edu/health/medical/ency/articles/ food-safety.

12. Willows ND. Determinants of healthy eating in Aboriginal peoples in Canada: The current state of knowledge and research gaps. Can J Public Health. 2005;96 Suppl 3:S32-6, S36-41.

13. Godin K, Stapleton J, Kirkpatrick SI, Hanning RM, Leatherdale ST. Applying systematic review search methods to the grey literature: A case study examining guidelines for school-based breakfast programs in Canada. Syst Rev. 2015;4:138.

14. Ontario Public Health Libraries Association [Internet]. Custom search engine for Canadian public health information. 2016 [consulté le 8 juin 2016]. Disponible à l'adresse : http://www.ophla.ca/customsearchcanada.htm.

15. Kraft C, Wuerz T, Cram J, Seaman L. Botulism presenting as dyspnea and respiratory failure in the Canadian Arctic. CMAJ. 2016;188(12):896-8.

16. Leclair D, Fung J, Isaac-Renton JL, Proulx JF, May-Hadford $\mathrm{J}$, Ellis A, et al. Foodborne botulism in Canada, 1985-2005. Emerg Infect Dis. 2013;19(6):961-8.

17. Pardhan-Ali A, Wilson J, Edge VL, Furgal C, Reid-Smith R, Santos $M$, et al. Community-level risk factors for notifiable gastrointestinal illness in the Northwest Territories, Canada, 1991-2008. BMC Public Health. 2013;13:63.

18. Larrat S, Simard M, Lair S, Belanger D, Proulx JF. From science to action and from action to science: The Nunavik Trichinellosis Prevention Program. Int J Circumpolar Health. 2012;71.

19. Toshach S. Brucellosis in the Canadian Arctic. Can J Public Health. 1963;54:271-5.

20. Proulx JF, Milor-Roy V, Austin J. Quatre éclosions de botulisme dans la régioin de la baie d'Ungava, au Nunavik, Quebéc. Relevé des maladies transmissibles au Canada [Internet]. 1997;23(4):30-2. Disponible à l'adresse : http://www.collectionscanada.gc.ca/ webarchives/20071125161208/http://www.phac-aspc.gc.ca/ publicat/ccdr-rmtc/97vol23/rm2304fd.html. 


\section{EXAMEN}

21. Hauschild AH, Gauvreau L. Foodborne botulism in Canada, 1971-84. CMAJ. 1985;133(11):1141-6.

22. Bowmer FJ, Wilkinson DA. Botulism in Canada, 1971-74. Can Med Assoc J. 1976;115(11):1084-6.

23. Dolman CE. Human botulism in Canada (1919-1973). Can Med Assoc J. 1974;110(2):191-7 passim.

24. Stuart PF, Wiebe EJ, McElroy R, Cameron DG, Todd EC, Erdman IE, et al. Botulism among Cape Dorset Eskimos and suspected botulism at Frobicher Bay and Wakeham Bay. Can J Public Health. 1970;61(6):509-17.

25. Serhir B, MacLean JD, Healey S, Segal B, Forbes L. Éclosion de trichinose associée à des morses de l'Arctique dans le nord du Canada, 1999. Relevé des maladies transmissibles au Canada [Internet]. 2001;27(4):31-6. Disponible à l'adresse : http://www.collectionscanada.gc.ca/ webarchives/20071214041942/http://www.phac-aspc.gc.ca/ publicat/ccdr-rmtc/01vol27/rm2704fb.html.

26. Viallet J, MacLean JD, Goresky CA, Staudt M, Routhier G, Law C. Arctic trichinosis presenting as prolonged diarrhea. Gastroenterology. 1986;91(4):938-46.

27. Chan J, Baxter C, Wenman WM. Brucellosis in an Inuit child, probably related to caribou meat consumption. Scand J Infect Dis. 1989;21(3):337-8.

28. Orr P, Lorencz B, Brown R, Kielly R, Tan B, Holton D, et al. An outbreak of diarrhea due to verotoxin-producing Escherichia coli in the Canadian Northwest Territories. Scand J Infect Dis. 1994;26(6):675-84.

29. Rowe PC, Orrbine E, Ogborn M, Wells GA, Winther W, Lior $\mathrm{H}$, et al. Epidemic Escherichia coli O157:H7 gastroenteritis and hemolytic-uremic syndrome in a Canadian Inuit community: Intestinal illness in family members as a risk factor. J Pediatr. 1994;124(1):21-6.
30. Jin A, Martin JD. Hepatitis A among residents of First Nations Reserves in British Columbia, 1991-1996. Can J Public Health. 2003;94(3):176-9.

31. Harb J, Lem M, Fyfe M, Patrick D, Ochnio J, Dobson S, et al. L'Hépatite A dans l'intérieur-nord de la ColombieBritannique : une éclosion dans une collectivité des premières Nations. Relevé des maladies transmissibles au Canada [Internet]. 2000;26(19):157-61. Disponible à l'adresse : http://www.collectionscanada.gc.ca/ webarchives/20071226025844/http://www.phac-aspc.gc.ca/ publicat/ccdr-rmtc/00vol26/rm2619fa.html.

32. Santé Canada [Internet]. Eau potable et eaux usées. Ottawa: Santé Canada; 2016 [mis à jour le 28 sept 2016; consulté le 20 juil 2016]. Disponible à l'adresse : http://www.hc-sc.gc.ca/ fniah-spnia/promotion/public-publique/water-eau-eng.php.

33. Damman S, Eide WB, Kuhnlein HV. Indigenous peoples' nutrition transition in a right to food perspective. Food Policy. 2008;33(2):135-55.

34. Kuhnlein HV, Receveur O. Local cultural animal food contributes high levels of nutrients for Arctic Canadian Indigenous adults and children. J Nutr. 2007;137(4):1110-4.

35. Government of Nunavut. Serving country food in government-funded facilities and community programs. 2016. Disponible à l'adresse : http://www. nunavutfoodsecurity.ca/sites/default/files/news/Serving $\% 20$ country\%20food.pdf.

36. Parkinson AJ, Butler JC. Potential impacts of climate change on infectious diseases in the Arctic. Int J Circumpolar Health. 2005;64:478-86. 


\section{Annexe : Bases de données utilisées et stratégie de recherche}

Base de données utilisée :

1. PubMed

2. Scopus

3. Cumulative Index of Nursing and Allied Health Literature (CINAHL)

4. Bibliography of Native North Americans

5. Sociological Abstracts

Exemple de base de donnée - termes de recherche pour PubMed :

(gastrointestinal disease*[tw] OR gastrointestinal illness*[tw] OR gastroenteritis[tw] OR intestinal disease*[tw] OR enteric disease*[tw] OR enteritis[tw] OR enterocolitis[tw] OR foodborne disease*[tw] OR foodborne illness*[tw] OR food poisoning[tw] OR waterborne disease*[tw] OR waterborne illness*[tw] OR diarrhea[tw] OR dysentery[tw] OR amebiasis[tw] OR botulism[tw] OR brucellosis[tw] OR campylobacter[tw] OR cholera[tw] OR cryptosporidiosis[tw] OR cyclosporiasis[tw] OR giardiasis[tw] OR hepatitis $A[t w]$ OR legionellosis[tw] OR listeriosis[tw] OR shellfish poisoning[tw] OR salmonella infection*[tw] OR salmonellosis[tw] OR shigellosis[tw] OR trichinellosis[tw] OR trichinosis[tw] OR escherichia coli[tw] OR yersinia infection*[tw] OR yersiniosis[tw] OR typhoid fever[tw] OR paratyphoid fever[tw] OR gastrointestinal diseases[MeSH:noexp] OR gastroenteritis[MeSH] OR intestinal diseases[MeSH:noexp] OR enteritis[MeSH] OR enterocolitis[MeSH] OR foodborne diseases[MeSH] OR diarrhea[MeSH] OR dysentery[MeSH] OR amebiasis[MeSH] OR brucellosis[MeSH] OR campylobacter[MeSH:noexp] OR cholera[MeSH] OR cryptosporidiosis[MeSH] OR cyclosporiasis[MeSH] OR giardiasis[MeSH] OR hepatitis A[MeSH] OR legionellosis[MeSH] OR listeriosis[MeSH] OR salmonella infections $[\mathrm{MeSH}] \mathrm{OR}$ trichinellosis[MeSH] OR escherichia coli[MeSH] OR yersinia infections[MeSH]) AND ((inuit[tw] OR rigolet[tw] OR "first nations"[tw] OR metis[tw] OR cree[tw] OR ojibway[tw] OR oji cree[tw] OR nunavut[tw] OR yukon[tw] OR "northwest territories" [tw] OR "northern ontario"[tw] OR arctic[tw] OR subarctic[tw] OR inuits[MeSH] OR nunavut[MeSH] OR yukon[MeSH] OR "northwest territories"[MeSH])) OR ((Canada[tw] OR Canadian[tw] OR Canada[MeSH]) AND (aborgin*[tw] OR indigen*[tw] OR native[tw] OR natives[tw])))
Base de données de littérature grise et sites Web utilisés :

1. Un moteur de recherche Google personnalisé (intégrant les sites Web des agences de la santé publique et des ministères de la santé fédéraux et provinciaux du Canada)

2. Base de données ProQuest Dissertations and Thesis Global

3. Sites Web du gouvernement fédéral
a. Santé Canada
b. Agence de la santé publique du Canada
c. Agence canadienne d'inspection des aliments

Termes de recherche dans la littérature grise:

Recherche 1. Foodborne illness AND Aboriginals OR First Nations

Recherche 2. Foodborne disease AND Aboriginals OR First Nations

Recherche 3. Waterborne disease AND Aboriginals OR First Nations

Recherche 4. Waterborne illness AND Aboriginals OR First Nations

Recherche 5. Gastrointestinal illness AND Aboriginals OR First Nations

Recherche 6. Diarrhea AND Aboriginals OR First Nations

Recherche 7. Food safety AND Aboriginals OR First Nations

Recherche 8. Food poisoning AND Aboriginals OR First Nations

*Les huit stratégies de recherche ont été appliquées à chaque moteur de recherche et jusqu'à 50 résultats furent analysés pour chaque recherche (soit 400 résultats par moteur de recherche au total). 